\title{
Die Chrysopoeia des Giovanni Augurelli
}

Giovanni Augurelli ist unter den Renaissancedichtern des ausgehenden fünfzehnten und beginnenden sechzehnten Jahrhunderts einigermaßen bekannt; er lebte von 1456 bis 1524 in Norditalien, zwischen Rimini, Venedig und Treviso, und er veröffentlichte (neben einer Reihe italienischer Sonette) lateinische Oden, Sermones und jambische Dichtung nach Horaz, vor allem aber die nach Vergils Georgica gestaltete Chrysopoeia in 3 Büchern, welche 1515 in Venedig erschien. ${ }^{1}$ Inhalt ist, wie der Titel sagt, die Kunst des Goldmachens, der Literatur in Prosa vor allem aus dem vierzehnten und dem späteren sechzehnten Jahrhundert, weniger hingegen aus Augurellis Zeit gewidmet ist, vor allem auf Französisch und Latein.

An zentraler Stelle beklagt sich Augurelli darüber, wie schwer es ihm falle, die Wörter einer barbarischen Fachsprache an den Leser eindeutig weiterzugeben, ${ }^{2}$ da seine Quellen selbst dadurch Verwirrung stifteten, daß sie bewußt für einzelne Gegenstände eine Vielzahl an Benennungen anführten. Und er endet sein Werk mit dem Bekenntnis, er habe seinen Bericht aus einer Pforte irgendwie zwischen der elfenbeinernen und hörnernen herausgetragen, habe also - nach der gängigen Interpretation der Vergilstelle - bewußt auch in seine Darstellungen Lügen eingestreut. ${ }^{3}$ Hieraus könnte man konstruieren, daß angesichts des

1 Benutzt wurde die Ed. princ. Chrysopoeia lb. III et Geronticon lb., Venedig 1515. Es folgten nicht weniger als 21 Neuauflagen. Daß sie jedoch stark gefragt wäre, muß eingeschränkt werden: weniger ihrer selbst willen wurde sie nachgedruckt, vielmehr als notwendiger Teil bei Sammelausgaben zur Alchemie. Darüber hinaus aber wurde die Chrysopoeia ins Französische und Deutsche übersetzt: François Hambert: Les trois livres de la Chrysopoeia, Paris 1626 (=1549); Valentin Weigel (1533-1588): Gülden-Vließ, Hamburg 1716. Scaligers Kritik: Poet. 6,4 (S. 785): „Augurelli multa vidimus, Lyrica, Sermones, Chrysopoeiam, Iambica. Sane prae se fert egregiam animi aequabilitatem. parum potest, parum praestat, parum conatur. [...] Elaboratior ipsius Chrysopoeia. caeterum vix adeo spirat: ita languida omnia, ac pene emortua. trepidationis potius quam limae agnoscas vestigia.“ Paracelsus schätzte das Werk und fragt nach der allegorischen Botschaft beispielsweise der Nymphe ,Glaura'; hierzu der Kommentar des Leo Suavius: Theophrasti Paracelsi Philosophiae et Medicinae [...] Compendium, Basel 1568, hier: Scholia in De vita longa lb. 3 zu Glaura = Mandragora, S. 288-293.

2 Chrys. 2,318-321 (f. e4r): „Utrunque innumeris prisci involvere tenebris | Barbaraque occultis rebus coniungere verba | Haud dubii: centum statuerunt nomina, quorum | Quodcunque incertam signaret remque modumque.“ Er spezifiziert allerdings nicht, wie dies genau gemeint ist: Fachwörter oder Fremdsprache?

3 Chrys. 3,695-697 (f. k3r): „Qua data porta inter geminas, quae somnia servant. | Cornea nec nobis patuit, nec prorsus eburnea | Emissus cecini falsis insomnia verbis.“ Der Sinn ist nicht ganz klar. 
neuen, der Antike völlig fremden Gegenstandes mit Vorgängen wie der Destillation und dafür Glas-geblasenen Instrumenten wie der Retorte, mit dem Repertoire der Alchemie also ,der Humanist‘ vor einem unüberwindlichen Hindernis stand. Daß er sich deswegen nicht anders helfen konnte, als antike Kinderschuhe über die Füße eines Pubertierenden zu zwängen. Man könnte also annehmen, Augurelli habe seine Materie mit den dichterischen Gerätschaften der Antike (naturwissenschaftliches Wissen, poetische Vorbilder, klassisches Vokabular) bearbeitet und wäre dabei unweigerlich immer wieder an eine Grenze des Möglichen gestoßen: dort wo das Wissen der Antike den Realitäten der Gegenwart nicht mehr entsprechen konnte. Und schon bei einer ersten Durchsicht hätte man die Bestätigung darin gefunden, daß ein Großteil des angeblich aktuellen Wissens lediglich aus den Berichten des Plinius rekonstruiert wird, während die modernen Verfahren vernachlässigt oder gar unterschlagen werden, oder daß Augurelli gar Wissen vortäuscht, das in einen antikisierenden Mythos gekleidet, gleichwohl verschwiegen werden kann. Aber ein solches Ergebnis, so sehr es Erwartungen erfüllen würde, ist vorschnell und wird sich als falsch erweisen.

Vor allem in jüngerer Zeit ist dem Werk hinderlich gewesen, daß man Augurellis Lehrepos nicht so recht in ein Sub-Genre einordnen kann: schon im achtzehnten Jahrhundert von Tiraboschi wurde die Chrysopoeia als literarischer Spaß aufgefaßt, ${ }^{4}$ als allegorisches Epos dagegen im letzten Jahrhundert von Zweder von Martels. ${ }^{5}$ Beides hat bisher nicht überzeugen können, so daß Robert Weiss' Bemerkung aus den 60ern, bis heute fehle eine das Ganze vereinbarende

4 Girolamo Tiraboschi: Storia della Letteratura Italiana, Modena 1776, 6,2, S. 231. Sein Urteil übernommen von Georg Roellenbleck: Das epische Lehrgedicht Italiens im fünfzehnten und sechzehnten Jahrhundert, in: Beitrag zur Literaturgeschichte des Humanismus und der Renaissance (Münchener Romanistische Arbeiten, Bd. 43), München 1975, S. 123-125. Literarisch vergleichbar wären die - nur wenig später entstehenden - Capitoli Berneschi (Berni Rime 54: Capitolo in lode d'Aristotele).

5 Zweder von Martels: The Chrysopoeia (1515) of Ioannes Aurelius Augurellus and the importance of alchemy around 1500. In: Studi Umanistici Piceni 13 (1993), S. 121-130. Ders.: The Allegorical Meaning of the ,Chrysopoeia‘ by Ioannes Aurelius Augurellus. In: Acta Conventus Neo-Latini Hafniensis. Proceedings of the Eight International Congress of Neo-Latin Studies. Copenhagen $12 \mathrm{Au}$ gust to 17 August 1991. Hg. von Rhoda Schnur et al. Birmingham, New York 1994, S. 979-988. Ders.: Augurello's ,Chrysopoeia“ (1515): A Turning Point in the Literary Tradition of Alchemical Texts. In: Early Science and Medicine 5,2 (2000), S. 178-195. Beides überzeugt nicht: Satirische Passagen über das Unwesen der Alchemisten lassen sich leicht erklären als entweder Verurteilung eben der falschen Quacksalber an eben rechter Stelle im Epos oder, häufiger noch, als Mißverständnis heutiger Interpreten, denen die Gepflogenheiten der derzeitigen alchemistischen Literatur nicht hinreichend bekannt waren. 
Interpretation des Epos, auch jetzt noch gültig ist. ${ }^{6}$ Ich will sie hier nicht geben, glaube aber, daß das Problem überschätzt wird und wir es mit einem gewöhnlichen Epos in der Nachfolge Vergils zu tun haben, dessen Außergewöhnlichkeiten sich durch die literarische Tradition der Materie erklären lassen, die es behandelt.

Zumindest für Augurelli ist erklärtermaßen Vergil der poetische Führer, der, wie er zu Beginn des 2. Buches erklärt, ${ }^{7}$ ihn zu dem Musenquell leiten wird: das erinnert zwar zuerst an Dante, ist aber ganz klassisch im Sinne der Imitatio

6 Robert Weiss, DBDI 4 (1962) = http://www.treccani.it/enciclopedia/giovanni-aurelioaugurelli_\%28Dizionario-Biografico\%29/ (14. Mai 2019): „Manca una interpretazione risolutiva sul carattere della Chrysopoeia: che il poema sia allegorico parrebbe certo; incerto è invece il significato preciso dell'allegoria e se il poema non sia sopratutto un esercizio di virtuosità metrica, un tentativo di dar veste poetica ad una matena [in der digitalisierten Version dieses Artikels auf Treccani online steht tatsächlich „matena“, was wohl ein Fehler der Texterkennung ist, denn es sollte "materia“" heißen] oscura ed arida."

Hieraus jedoch (mit Zweder v. Martels, 1991) den Schluß zu ziehen, beim Werk handele es sich vielmehr um ein durchweg allegorisches, wie es der Fall für ein anderes, früheres von $\mathrm{Au}$ gurelli ist, das Vellum Aureum, geht - wie ich meine - zu weit: zwar finden sich durchweg allegorische Bilder, darunter auch neu gebildete; aber das Wichtigste für eine Allegorie der Alchemie im Rahmen einer neuplatonischen Weltsicht fehlt: nirgendwo wird behauptet, die Befreiung des Spiritus aus der Materie habe analoge Folgen für die Befreiung des Spiritus des Alchemisten. Auch wenn betont wird, daß der Alchemist ein ethisch einwandfreies Leben zu führen habe, ist dies nicht Vorbedingung zum Gelingen des Opus Magnum. Ganz im Gegenteil steht immer zu fürchten, daß ein moralisch Minderwertiger das Werk vollende und dem gemeinen Volk zur Kenntnis bringe. Insofern kann man die vielen praktischen Anweisungen des Epos nicht einfach als Digressionen eines eigentlich allegorischen Werkes subsummieren, und man muß fragen, warum es denn nicht möglich sein sollte, das Werk analog zu denen der klassischen Antike als tatsächlich der poetischen Vermittlung einer Techne Gewidmetes zu erklären?

7 Chrys. 2,8-11 (f. d3r): „Tuque ades, o cultum Musis et Apolline pectus, | Ante alios olim cunctos, divine poeta, | Quo duce secretum per iter procedere tantum | Ipse queam [...].“ Augurelli stellt sich damit nicht in die Nachfolge des Lukrez, obwohl er dessen Klage der schwierigen Übersetzung, auch die Präsenz physikalischer Ausführungen mit ihm teilt, sondern vielmehr der Georgica, deren Gegenstand ein ganz traditioneller war, welcher letztlich keine besondere Rechtfertigung erforderte. Von ihrer Struktur übernimmt Augurelli die Zweiteilung seiner Bücher: das erste teilt sich in die Theorie der Alchemie und die praktische Verwirklichung durch Adepten; das zweite in die falsche und richtige Vorgehensweise des alchemistischen Prozesses. Das dritte schließlich kann grob in die Herstellung des Elixirs zuerst, dann die der Tinctura eingeteilt werden; es geht aber darüber hinaus. Hier findet sich ein längerer Exkurs über Nebenprodukte, die Alchemisten bei ihrer Suche entwickelten: der Titel Chrysopoeia scheint damit nicht mehr zu korrespondieren. Aber auch der auf die Tätigkeit des Landwirts sich beziehende Titel Georgica ist, spätestens wenn es um die Bienen geht, zu eng gefaßt, um das Ganze zu repräsentieren: Augurelli bleibt also in den Fußstapfen seines Vorbildes. 
zu verstehen. Am Beginn seines dritten Buches erwähnt Augurelli, daß, nachdem Vergil die Natur oberhalb der Erde poetisiert habe, er sich dagegen der Natur unterhalb der Erde widme. ${ }^{8}$ Ich halte es insofern für eindeutig gesichert, daß eine Erklärung der Chrysopoeia nur innerhalb der Parameter einer klassischen, durch die Georgica vorgegebenen Tradition möglich ist; für die Georgica sind aber zu Augurellis Zeiten weder Erklärungen als satirisches noch allegorisches Werk belegbar. Hieraus folgt, ganz unabhängig davon, wie hoch ein satirischer bzw. allegorischer Anteil sein mag, daß auch die Chrysopoeia grundsätzlich dem poetischen Darstellen, im Anspruch nach auch dem wirklichen Vermitteln einer Fertigkeit gewidmet ist.

Dies ist eine erste Erklärung für das Vermeiden von alchemistischen Fachbegriffen, auch wenn alchemistische Praxis beschrieben wird: wer den älteren Cato, Varro und vor allem Columella mit Vergil vergleicht, sieht unschwer die Forderungen, die eine Poetisierung an die Realität stellt: kaum ist in den Georgica die doch unverzichtbare Rolle der Sklaven thematisiert; gar nicht wird - vor lauter Ulmen, von denen Trauben hängen - die doch gängige Praxis der Rebenstöcke erwähnt; das Konservieren der Ernte für den Winter, das Anlegen eines Gartens, Rechenschaftsberichte eines Vilicus, Steuerabgaben von Pächtern - all dies weicht, wohl als unpoetisch, einem Buch über den Bienenstaat mit mythologischem Epyllion. Tatsächlich werden Fertigkeiten behandelt, aber eben mit poetischen Mitteln, die mehr den Kern der Fertigkeit freilegen als sich in Details zu verlieren, deren Aufzählung einem Prosahandbuch vorbehalten bleiben sollte. Unter dieser Gleichsetzung bräuchte es tatsächlich keiner anderen Begründung für das Verfassen der Chrysopoeia: wie viele andere zeitgenössische Lehrepen wirbt sie für eine Fertigkeit, die derzeit real in die Tat umgesetzt wurde.

Allerdings kann Augurelli ein Lehrepos im Sinne einer De Re Metallica weder verfassen noch ein solches verfaßt haben wollen, da ihm elementare Kenntnisse der Mineralurgie tatsächlich zu fehlen scheinen. ${ }^{9}$ Das Wissen um die Fundorte der

8 Vgl. Chrys. 3,16-22 (f. g2v): „Extremam tantorum operum ne desere curam | Hanc nostram, oramus: nec, si quae maximus olim | Ille tuus cecinit vates ornantia supra | Tellurem complexa foves, contemnere prorsus | Inducas animum, quae nos imitamur, et ipsis | Illius impressis quae nunc vestigia signis | Ponimus internae scrutati viscera terrae.“

9 Anstelle solcher Beispiele, welche einen Leser der Chrysopoeia bestimmt beeindruckt hätten, behilft sich Augurelli im zweiten Buch mit dem Mythos des Lynkeus, der kraft seiner Augen die Innereien der Erde durchschauen konnte. Dies ist zwar mit dem Mythos vereinbar, nicht jedoch Gegenstand des antiken Mythos selbst gewesen. Dem Lynkeus bereitet Arethusa dann auch einen Seesturm zur Strafe, daß er mit seinem Superblick bei den Nymphen gespannt hatte, aber erreicht damit nichts, denn weiter schaut sich Lynkeus die Gebirgsformationen von innen an. Das Resultat ist ein irritierter Leser, der sich mehr erwartet hatte und beim besten Willen auch keine allegorische Erklärung in der Erwähnung dieses Lynkeus finden kann. 
Metalle erklärt Augurelli mythologisch mit dem Wirken des Argonauten Lynkeus, der sie dank seines Scharfblickes kartographieren konnte; den Fundort des Urmetalls lokalisiert er bei der Grotte der Nymphe Glaura, deren Betreten allerdings die Trennung von Seele und Leib, den Tod, zur Folge haben würde. ${ }^{10}$ Der lange Exkurs zum Abbau von goldhaltigem Gestein im zweiten Buch ist fast wörtlich aus der Naturalis Historia des Plinius gezogen; ${ }^{11}$ die dort geschilderte Technik wurde längst nicht mehr angewendet. ${ }^{12}$ Einerseits hatte der Silberbergbau untertage in der Nähe von Innsbruck bei Schwaz zu Augurellis Zeit immense Fortschritte gegenüber der Antike gemacht; ${ }^{13}$ andererseits war der Goldbergbau etwa in den zu Salzburg gehörigen Hohen Tauern, dessen Gold Ende des fünfzehnten Jahrhunderts nach Venedig exportiert wurde - bei weitem nicht so aufwendig, wie bei Plinius für Las Médulas beschrieben, sondern beschränkte sich auf kleine Stollen unterirdisch und oberirdische Goldwäschereien durch die ansässigen Bauern. ${ }^{14}$ Auch zum Tauchen nach Perlen wiederholt Augurelli

10 Der Name ist vielleicht mit Augurellis Freund zu erklären, dem Humanisten Pomponius Gauricus (1481/82-1530), oder dessen Bruder Luca (1476-1558), beide geboren in Gauro, seit 1501 Studenten in Padua und mit astrologischen bzw. alchemistischen Interessen; die Mutation des Namens in Glaura erklärte sich dann als (petrarkistisches) Spiel mit Begriffen wie: Gloria/Aurum bzw. Laurus/Laura/L'oro.

11 Plin. nat. hist. 33,70-78.

12 Allerdings weiß Augurelli offensichtlich Neues vom Donaugold: Chrys. 2,302 ff. (f. e4r): „Ut Istri | Accola felicis saepe illud stirpibus herens | Capreoli in morem nati circundare truncos: | Implicitumque etiam saxis invenit obesis.“

13 Franz-Heinz Hye: Stadt und Bergbau in Tirol mit besonderer Berücksichtigung der Städte Hall und Schwaz, in: Das kulturelle Erbe in den Montan- und Geowissenschaften. Bibliotheken - Archive - Sammlungen. 8. Internationales Symposium vom 3. bis 7. Oktober 2005 in Schwaz. Geschichte der Erdwissenschaften in Österreich. 5. Arbeitstagung vom 3. bis 7. Oktober 2005 in Schwaz. Hg. von Tillfried Cernajsek, Wien 2005 (Berichte der Geologischen Bundesanstalt 65), S. 81-89; Gert Amman (Hg.): Silber, Erz und weißes Gold. Bergbau in Tirol. Tiroler Landesmuseum Ferdinandeum (Ausstellungskatalog. Tiroler Landesausstellung in Schwaz, Franziskanerkloster und Silberbergwerk, 20. Mai bis 28. Oktober 1990), Innsbruck 1990.

14 Fritz Gruber: Der Edelmetallbergbau in Salzburg und Oberkärnten bis zum Beginn des 19. Jahrhunderts, in: Schatzkammer Hohe Tauern. 2000 Jahre Goldbergbau. Hg. von Wilhelm Günther, Werner Paar, Salzburg 2000, S. 261f. Die Fugger aus Augsburg hatten 1484 sowie 1489 die ,Kammer der Judenburger' im ,Fondaco dei Tedeschi“ in Venedig inne; Salzburger Gold wurde in diesem Zeitraum nach Venedig verkauft: Karl-Heinz Ludwig: Gold und Edelmetall in der europäischen Montangeschichte unter besonderer Berücksichtigung des Erzstifts Salzburg, S. 89-112, hier S. 102; der Verkauf nach Venedig endete 1501: Christoph Mayrhofer, Peter F. Kramml: Empfang ich aus der Minz ... Zu Münzprägung und Bergbau in Salzburg von 1400 bis 1600, S. 157-167, hier S. 160, beides in: Das Tauerngold im europäischen Vergleich. Archäologische und historische Beiträge des Internationalen Kongresses in Rauris vom 7. bis 9. Oktober 2000. Hg. von Gerhard Ammerer, Alfred S. Weiß, Salzburg 2001. 
,seinen Plinius‘ selbst dort, wo er vom ominösen Muschelkönig schreibt; ${ }^{15}$ über den Export und Import indischer Perlen erfährt der Leser allerdings nichts, noch viel weniger von etwaigen Perlen aus der Neuen Welt. Von der Gewinnung der Rohstoffe hatte Augurelli also keine über das Oberflächliche hinausgehende Kenntnis; er hatte auch offensichtlich kein Interesse, sein aus Plinius gewonnenes Wissen $\mathrm{zu}$ aktualisieren.

Dagegen kann er sich in der Vier-Elemente-Theorie sicher bewegen und auch innerhalb der Alchemie eine bestimmte Lehre gegen andere vertreten. Dies ist Gegenstand der jeweils ersten Hälften des ersten wie zweiten Buches: Augurelli weist in einem theoretischen Teil zuerst nach, daß auf dem Boden der derzeit gültigen Physik eine Transformation der Elemente denkbar ist; er nimmt einen Spiritus auch in den mineralischen Dingen an, der durch alchemistische Behandlung eine Fortpflanzung vollziehen kann; er nennt Beispiele für unglaubliche Transformationen, etwa aus dem Tierreich die Metamorphose der Raupe zum Schmetterling. Im zweiten Buch verfolgt Augurelli zuerst teils mit satirischer Ader die gescheiterten Versuche, Alchemie unter Verwendung abseitiger Materialien zu betreiben; er setzt sich dagegen für das Prinzip des Simile ein, also die Gewinnung und Bearbeitung eines verwandten, aber philosophischen Urmetalls: während alchemistische Autoritäten wie Ps-Lullus hierin den Mercurius, das Quecksilber, identifizieren, nennt Augurelli das Gold selbst. Dies aber ist nur eine Frage des Vokabulars, denn gemeint ist nicht das uns bekannte Element, sondern sein philosophisches Gegenstück, das Urmetall.

Trotzdem hat man - besonders im gewesenen Jahrhundert - ihm unterstellt, daß er vermutlich nie das Studio eines wirklichen Alchemisten gesehen haben mag, und zeugt die berühmte Anekdote, auf seine Widmung habe er vom Papst eine leere Börse erhalten, daß es ihm auch bei einer Vielzahl von zeitgenössischen Lesern nicht gelungen ist, sich als Sachverständigen zu profilieren. Dies liegt nicht daran, daß es ihm an Quellen mangelte: neben Ps-Lulls ${ }^{16}$

\footnotetext{
Zum Tauerngold auch: Wilhelm Günther, Werner Paar: Schatzkammer Hohe Tauern, Salzburg u. München 2000. Das Versiegen der Goldquelle Venedigs 1501 könnte mit ein Motiv für Augurelli gewesen sein. Weiter von Augurelli erwähnt wird Seifengold aus dem Donaugebiet, Chrys. 2,303-306: „[...] ut Istri | Accola felicis saepe illud stirpibus haerens | Capreoli in morem nati circundare truncos, | Implicitumque etiam saxis invenit obesis." Ungarngold war im Rahmen der europäischen Produktion der bedeutendste Sektor im sechzehnten Jahrhundert.

15 Plin. nat. hist. 9,104-124, hier 111: „quidam tradunt sicut apibus, ita concharum examinibus singulas magnitudine et vetustate praecipuas esse veluti duces, mirae ad cavendum sollertiae."

16 Raimundi Lulli Majoricani Testamentum ultimum angelorum [...] aus dem Digitalisat: BSB Clm 10493, Druck ohne Jahr (siebzehntes bis achtzehntes Jahrhundert). Augurellis Behauptung, mit einem Korn einen ganzen Ozean tingieren zu können (3,670-674): „Illam
} 
Testamentum Ultimum Secretum Angelorum ist es sogar eine lokale Größe aus Treviso, die nach jüngerer Forschung zwei verschiedene Personen repräsentiert: Bernard oder Bernhard von Trevisan, der - wenn man sie als eine Person liest - ebenfalls die These aufgestellt hatte, daß Gold sich lediglich durch ein Simile, den philosophischen Mercurius, herstellen lasse. ${ }^{17}$ Zumindest von der

adeo interdum priscis auctoribus auctam, | Ipsius ut tenui proiecta parte per undas | Aequoris, argentum si vivum tum foret aequor, | Omne vel immensum verti mare possit in aurum.“ Vergleiche mit Ps-Lull, Test. Ult. 4,9 (S. 141B): „Si igitur acceperis unam parvam guttam de illo sive albo sive rubeo ad quantitatem unius grani cannabis vel ciceris, et etiam, quantum et granum milii et illum proieceris super millies mille partes argenti vivi vulgi, dico, quia illum transmutabit in aurum et argentum secundum qualitatem medicinae melius minerali ictu oculi sine ignis iuvamento, sed solo odore illius, et facit, sicut oculus basilisci aut fulgur, quod omnia permutat in istud, et permeat in profundum, sicut aliquando visum fuit consumpsisse nummos in crumena sine laesione illius, aut ensem vagina illaesa contrivisse, sic iste carbunculus penetrat in profundum mercurii et metallorum et illa convertit in suam naturam solo tactu et odore. [...]“ Im Folgenden behauptet er, dies selbst in Anwesenheit des englischen Königs getan zu haben, und geht dann über auf den Bau des Alchemistenofens (wie Augurelli die Cucurbita beschreibt). Das Werk ist nur handschriftlich überliefert, und zwar von der Mitte des fünfzehnten Jahrhunderts an und schwerpunktmäßig in Florenz zu finden. Auch untersucht, aber ohne Ergebnis: Bibliotheca Chimica Bd. I: Testamentum 707-762; Pars Practica 763-777; Novissimum: 790-805.

17 Zur Person: Joachim Telle: Lexikon d. Mittelalters 1 (1980), Sp. 2005-2006; im Folgenden wird keine Trennung der beiden Autoren gemacht, da mutmaßlich Augurelli eine solche nicht bewußt war. Text aus: Johann Jacob Mangetius: Bibliotheca Chemica Curiosa, Genf 1702, Bd. 2, hier: Bernard von Trevisan, Liber de Secretissimo Opere Chemico ... item Responsio ad Thomam, S. 388-399 u. 399-409. Zur Ablehnung einer Vielzahl von Ingredientien: Bern. Trev. De Secretissimo, Bd. 2, 392A: „Suadeo, ut alumina, vitriola, salia et atramenta omnia, boraces, aquas fortes, animalia et omne quod ex eis provenit, crines, sanguinem, spermata, carnes, ova, lapides, et mineralia quaeque relinquas, pariter et metalla sola." Zur daraus abgeleiteten ,Nur-Quecksilber'-Lösung (S. 393A über den Spiritus): „His verbis itaque Geber concludit, ad hunc pretiosum lapidem nullo alio opus esse, quam sola substantia Mercurii per artem optime mundati, penetrantis, tingentis, stantis in praelio adversus ignem, et ni diversa separari se non permittentis, ac semper in sola sua Mercuriositatis substantia constantissime persistentis, tum demum (inquit) coniungitur metallis in profundo radicaliter, formam ipsorum corrumpendo ac aliam introducendo iuxta virtutem et colorem elixiris, aut medicinae tingentis." Daß Augurelli Ps-Geber also selbst gelesen habe, ist - wie diese Stelle zeigt - nicht zwingend, da sich seine Quellen namentlich auf Geber als den „Erz-Alchemisten“ bezogen haben konnten. Zur Wirkung des Steins: Bernard, De Secretissimo 390B: „Corpore vulgaria, per naturam solam in mineris absoluta, sunt mortua, ut imperfecta perficere nequeant, verum si per artem ad septenam, denam vel duodenam suae perfectionis multiplicationem adducantur, eatenus tingunt ad infinitum [...].“ So auch der Autor der Responsio, Bernard, Ad Thomam responsio, Bd. 2, S. 399B: „Similiter argentum vivum est omnium metallorum materia.“ Daß der Alchemist bewußt Falsches schreibe, siehe unter anderem in Bernard, Responsio, 407B: „Oportet autem te intelligere quod Geber summa prudentia et miro artificio veritatem sub velamine 
jüngeren dieser beiden Personen, deren Werke auf Französisch wie Lateinisch überliefert sind, scheint die Biographie eine Fiktion zu sein, denn sie soll 1452 Italien für eine umfangreiche alchemistische Reise verlassen haben; ihr soll dann - nachdem alle Versuche jämmerlich mißlangen - 1481 das Magisterium auf Rhodos gelungen sein, wo sie 1490 gestorben sein soll. Der Gedanke ist verführerisch, daß Augurelli hier genauere Informationen gehabt haben könnte, und daß der fiktionale, teils satirische Charakter der Autobiographie zu den satirischen Elementen in der Chrysopoeia beigetragen habe.

So mit der Theorie ausgestattet, hätte es Augurelli eigentlich gelungen sein müssen, die Wirksamkeit der Alchemie wahrscheinlich zu machen, zumal er im zweiten Teil des ersten Buches auf Adepten verweisen kann, denen die Transformation in Gold tatsächlich gelungen wäre: er nennt den Erzalchemisten, Raimundus Lullus, dem 1380 - und damit natürlich postum - das Magisterium in Mailand gelungen sein soll; aus dem eigenen Umfeld Padua kann er möglicherweise aus Experimenten des postum mit dem Tode bestraften Petrus von Abano zitieren: zerbrochene Gefäße als Relikt einer gelungenen Transmutation zu Silber. Und schließlich verweist er auf ein alchemistisches Experiment, an dem er persönlich teilgenommen hätte. ${ }^{18}$

Von der Theorie der Alchemie hat Augurelli also Kenntnis; auch scheint er bei zumindest einem Experiment zugegen gewesen zu sein; den ganzen Ablauf hat er aber - naturgemäß - nicht verfolgen können und kann ihn deswegen auch nicht anschaulich berichten. Die alchemistischen Werkzeuge jedoch kennt er und widmet ihnen gelegentliches Interesse: im zweiten Buch erklärt er den Aufbau und die Wirkung des sogenannten „Pelikan“, eines Kolbens zur zirkulierenden Destillation, der hierfür zwei hohle Henkel aufweist. Im dritten widmet er sich dem vierteiligen Set zur gewöhnlichen Destillation, das - im Unterschied zur Retorte - eine gesonderte Halbkugel oder Helm mit Tülle, Abflußrohr oder Schnabel namens Alembik aufweist, die den Dampf aus dem nach dem Kürbis

occultat, multas obscuritates aut falsitates intermiscens, quas inscii prima fronte existimant veritatem esse [...].“ Das Verbergen und Mitteilen alchemistischer Informationen unter allegorischem Mantel findet sich bei Bernard, De Secretissimo, 397B-398B, wo die fiktive Erzählung des Autors, welcher sich an einer Quelle findet und auf den sog. Goldenen König wartet, ganz ähnlich zu der Glaura des Augurelli wirkt. Die Passage konnte auch eine mögliche Inspiration zur Verfassung des Einzelgedichts Vellus Aureum gewesen sein, das sich bereits unter den 1505 in Venedig veröffentlichten Gedichten Augurellis findet.

18 Chrys. 1,557-571. Petrus d'Apona/de Abano 1250/57-1316, seit 1306/07 an der Univ. Padua Lehrstuhl für Medizin. Die Annahme, er habe reges Interesse an der Alchemie gezeigt, stützt sich allerdings wohl nur auf den Vorwurf der „Magie“, der ihm in den Anklagen von 1300 und 1306 gemacht wurde. 
Cucurbit genannten Kolben auffängt und weiterleitet zum Auffanggefäß. Ihn interessiert dabei besonders das Material des Cucurbiten, welches die hohen Temperaturen des Alchemistenofens Athanor aushalten muß. Der hier zu verwendende Stoff setzt sich nach Augurellis offensichtlich unvollständiger Liste zusammen aus mindestens spanischer Soda, welche Venedig zur Glasherstellung importierte, weißem Ton und damit möglicherweise Kaolin, Fango das heißt Naturlehm aus dem Fluß Abano bei Padua, und schließlich vermutlich Alabaster. Hieraus läßt sich Glas herstellen, wie es bei vielen noch erhaltenen alchemistischen Kolben auch dokumentiert ist, ${ }^{19}$ aber auch Fritte, wie man sie für Keramik wie Majolika und Fayence braucht. ${ }^{20}$ Weiter interessiert Augurelli ein Präzisionsmeßgerät, das sogenannte Templum: es handelt sich um eine Goldwaage, die der Reinlichkeit halber in eine Glasvitrine eingeschlossen wird. Soweit die Instrumente. An den Ingredientien, den Reaktionsmaterialien interessiert Augurelli vor allem, daß traditionelle Zutaten wie Wein, Eier, Kräuter, auch solche zur Reinigung des Schwefels wie Natrum, Salze, auch Aquavit überflüssige, ja verunreinigende Beitaten sind, auf die man verzichten könne. Hierin folgt er der Simile-Theorie des Bernard von Treviso, und zwar gerade der satirischen Episode seiner Autobiographie, in welcher vergebens Versuche mit den vermeintlich absurdesten Materialien, darunter Menschenblut und Urin, erwähnt werden. ${ }^{21}$

Durchaus aber hätten, so Augurelli nun neu, Produkte wie der Tresterschnaps einen Eigenwert, nur hülfen sie nicht bei der Goldtransformation. Ferner interessieren ihn auch Stoffe und Produkte, die nicht direkt zur Goldtransformation dienen, sondern zur gewöhnlichen Goldgewinnung: neben dem eher fabulösen Merkurialwasser etwa das zur Trennung von Silber und Gold entwickelte Scheidewasser und das Auripigment, aus welchem laut Plinius Caligula habe Gold herstellen wollen, ${ }^{22}$ das gewöhnlich aber in der Malerei als Goldfarbe verwendet wird. Wenn auch nur als Gleichnis, so interessiert ihn auch das sogenannte Safrangelb, da es die Wirkung des Philosophensteins illustrieren könne, mit wenig Ausgangsmaterial - hier also dem gelbfarbenen Safran - eine ungeheure Menge zu tingieren - hier natürlich neben dem einfachen Wasser die Lösung in Eigelb oder Öl zum Farbpigment

19 Dazu https://www.archaeologie-online.de/artikel/2008/alchemistische-gefaesse/ (besucht 03.06.2019): Peter Kurzmann: Aus der Küche ins alchemistische Laboratorium.

20 Zum Dichtbrennen war beim Niedrigbrand eine Lasur nötig; dies war die Fritte. Zu Venezianer Glas aus archäologischen Funden vgl. Carl Pause: Spätmittelalterliche Glasfunde aus Venedig, Bonn 1996.

21 Wie Anm. 17, Bernard, De Secretissimo, Bd. 2, 392A; Dazu. Chrys. 2,42-44. „Nec varias specie res admiscebit, et uni | Quodcunque obstiterit iunctum, procul inde movebit. [...]“ 48-50: „Quid levis humano missus de corpore sanguis? | Quid lapsi e rosea flavi cervice capilli? | Ova quid et lectae summis in montibus auro?“

22 Plinius nat. hist. 33,4. 
Safrangelb. ${ }^{23}$ Und - ganz bemerkenswert - interessiert ihn auch die Möglichkeit, den Prozeß des Dicklegens von Milch ohne Lab in Gang zu setzen: die Beschreibung paßt möglicherweise auf die Herstellung von Ricotta oder Zigher, Frischkäse, die durch Erhitzung von Süßmolke unter Beimischung von Milch gewonnen werden. ${ }^{24}$

Augurelli kümmert sich also auffällig um Verfahrenstechniken, Instrumente, Industrien; sie sind keineswegs zufällig. Nicht die Silberbergwerke von Schwaz noch die Goldminen der Hohen Tauern finden sein Interesse, sondern jene Industrien, die zu seiner Zeit im Dreieck zwischen Rimini, Venedig und Treviso europaweit führend ausgeübt wurden. Grappa, Glas, Farbpigmente dies waren Schlüsselbereiche der Venezianer Technologie, die noch den Untergang des Kolonialreiches überlebend im neunzehnten Jahrhundert führend blieben. ${ }^{25}$ Und so ist es auch für die folgenden Beispiele, die nicht mehr eng mit der Goldtransformation in Verbindung stehen.

Bekanntlich dient das Elixier nicht nur als Universalmedizin, sondern wäre auch in der Lage, mattgewordene Perlen wieder zu verjüngen. Hiervon ausgehend widmet sich Augurelli im dritten Buch lange der natürlichen Gewinnung von Perlen, um über ihre künstliche Gewinnung durch das Elixier schließlich auf Glas- und vermutlich auch Schmelzperlen überzugehen. Venezianer Perlen waren ein hochgefragtes Produkt, das insbesondere im Afrikahandel Verwendung fand; die Insel Murano war Ort ihrer Herstellung, welcher von den Produzenten nicht einfach verlassen werden konnte, so geheim waren die Prozesse. Die Universalmedizin hingegen, Objekt so vieler Hoffnungen, interessierte Augurelli nicht.

Daß Venedig als Seemacht Interesse an nautischem Wissen hatte, erklärt wohl die lange Erwähnung des Magnetsteins, gar des sagenumwobenen Magnetbergs; vielleicht allerdings auch, weil er nach Georg Agricola zu den Rohstoffen des Kristallglases zählen sollte. ${ }^{26}$ Den Kompaß und die Permanentmagnetisierung,

23 Chrys. 2,492ff.: „Utque croci exiguus pura flos sparsus in unda | Immodicum grato diffundit odore colorem | Plurima sic agitans contracto in pulvere virtus | Obvia percurrit liquidi loca cuncta Metalli.“

24 Chrys. 2,475-479: „Ac veluti modicus, si forte coagula desint, | Caseus extemplo lympha resolutus et igni | Adstringit plena liquidi mulctraria lactis, | Cogit et alterius lac in se vertere formam: | Sic argenta sacer pulvis liquentia sistet.“ Zur Erklärung nehme ich an, ,modicus caseus lympha resolutus' bedeute Molke = Serum.

25 Hier allgemein: Gino Luzzatto, Storia economica di Venezia dall’XI al XVI secolo, Venedig 1961, Nachdruck 1995. Franco Brunelli: Arti e mestieri a Venezia nel medioevo e nel rinascimento, Vicenza 1981.

26 So Georg Agricola, De re metallica 12, Ende; der Gedanke nach Plinius 36,66. Tatsächlich wurde Braunstein (Pyrolusit) verwendet. Kompaß = klappbare Sonnenuhr, die einen Kompaß 
eigentlich ein für sein Vorhaben analoges Verfahren, nennt Augurelli ebenfalls nicht: geschah dies, weil Zentrum dieser ebenfalls jungen Technologie Nürnberg war und nicht Venedig? Daß Augurelli jedenfalls die Verwandlung in Gold mit der Metamorphose eines Schmetterlings vergleicht, ist nachvollziehbar; daß er aber statt eines europäischen Schmetterlings ausgerechnet die Seidenraupe bombyx nennt, erklärt sich nur über die Venezianer Seidenindustrie, die soeben in einem Lehrgedicht des mit Augurelli bekannten Marco Girolamo Vida gefeiert wurde. ${ }^{27}$ Auch für die Glasherstellung der Antike interessiert er sich, allerdings im Rahmen des Verbots der Weitergabe von Technologie: wie Tiberius den Erfinder eines unzerbrechlichen Glases hat vorausschauend umbringen lassen, ${ }^{28}$ und wie Diokletian einen Feldzug nach Ägypten führte, um alchemistisches Wissen zu unterdrücken. ${ }^{29}$

Und schließlich die bildende Kunst: im Sinne der Metallkunde ist es naheliegend, einen Bronzegießer hervorzuheben, der mit vermutlich alchemistischer Hilfe besondere Resultate erzielt. Augurelli nennt Andrea Briosco il Riccio, einen lokal bekannten Künstler, und vergleicht ihn mit dem antiken Myron. ${ }^{30}$ Aber kein Detail deutet auf eine direkt mit der Alchemie verbundene Innovation. Auch die Kunst, Bronzekanonen zu gießen, scheint er wichtiger zu nehmen als das - im Rahmen alchemistischer Nebenprodukte erwähnenswerte -

enthielt. Hier vgl. Friedrich Bradhering: Kurze Geschichte des Schiffskompasses. In: Sechzehnter Jahresbericht [...] zu Magdeburg, Magdeburg 1902; Die Kompaßmacher aus Nürnberg und Fürth, die Gilde der Kompaßmacher wurde allerdings erst 1510 gegründet; Erhard Etzlaubs (1460-1532) erste erhaltene Uhr datiert auf 1511. Aus Nürnberg stammende Sonnenuhren waren auch in Italien begehrt; Venedig war dagegen kein Zentrum der Produktion.

27 Nach dem aus Verg. Georg. 4 gezogenen Beispiel mit dem Stieropfer für den Bienenstaat dann sofort Chrys. 1,183-187: „Multus tenero sub pectore bombix | Palpitat et lecta nutritus fronde virentis | Dehinc mori, tenuis texto se velleris orbe | Implicat, ac tandem pedibus munitus et alis | Papilio de verme pedes volitansque recedit.“ Vida hatte Augurelli in mehreren Gedichten gelobt und war umgekehrt von ihm auch dichterisch gelobt worden. Sein De bombyce entstand um 1505.

28 Chrys. 3,450-452: „Caverat hac princeps re forsan Claudius una | Flexibile ut fieret mirando examine Vitrum: | Detraheret nequid precia olim imposta Metallis.“ Gemeint ist Tiberius Claudius Caesar; die Nachricht aus Plinius nat. hist. 36,195.

29 Siehe: Chrys. 3,453-457: „Vidit idem inferior longo post tempore Caesar | Ipse etiam fortasse, artis cum perdere tantae | Artifices voluit, iussitque exurere librum | Multorum aggestum cumulum, dum forte peragrat | Milite composito Aegypti ditissima regna: | [...].“ Quelle ist hier ,Suidas' s.v. Chemeia.

30 Plin. nat. hist. 36,32: berühmt wegen seiner Bronzen; Brios(c)o 1479-1532, geb. Trento, lebte in Padua; war besonders berühmt für kleine Bronzen und Gemmen, die antike Themen aufnahmen oder sogar neu verwirklichten. Von besonderer Schmelztechnik, etwa Vergoldung durch Brennen, habe ich allerdings nichts in Erfahrung bringen können. 
Pulver selbst, geht aber wiederum nicht in Details. Weiter zu führen scheint die Erwähnung eines Malers, Giulio Campagnola, der uns heute vor allem als innovativer Kupferstecher durch seine Sticheltechnik bekannt ist. Dieser Giulio Campagnola könne, nach Augurelli, mit Farben jede mögliche Wirkung erzielen; im Folgenden beschreibt er die Herstellung eines künstlichen, blauen Farbpigments zum Ersatz der Rohstoffe Lapislazuli oder Azurit. Die Herstellung künstlicher Pigmente lag in den Händen von Spezialisten, zumindest in einer Stadt wie Venedig; umgangssprachlich wurden sie als Alchemisten bezeichnet. Bei Vasari heißt es deshalb in aller Selbstverständlichkeit, ${ }^{31}$ es gäbe zwei Arten von Farbpigmenten: „i quali son fatti parte da gli alchimisti e parte trovati nelle cave.“ Dies hat in der Forschung zur Annahme geführt, Augurelli hätte für Campagnola persönlich Pigmente für die Farbe Blau hergestellt; ${ }^{32}$ im Unterschied zur Goldgewinnung, die er nach Plinius beschreibt, müßte Augurelli deswegen um detailliertes Wissen zur Herstellung von Farbpigmenten verfügt haben. Leider ist diese Verbindung vorschnell konstruiert worden: Augurelli sagt nirgendwo, daß Campagnola dieser speziellen Farbe besonders bedürftig sei, wenn er die Herstellung seines meerblauen Pigments beschreibt, und sein Pigment ist dazu noch alles andere als innovativ.

Ein intensives Blau ist die ärgerlichste Farbe der Malkunst für lange Zeit gewesen; das hierfür benötigte Pigment gehörte zu den Grundstoffen, die der Auftraggeber zur Verfügung stellen mußte; Lapislazuli und Azurit aber waren immens teuer. ${ }^{33}$ Augurelli rät deshalb zu einem Pigment, das künstlich hergestellt werden muß, indem man von flavum aurum, also von Gold, kleine Späne abschabt, sie in Essigsäure fallen und dieses dann in organischem Abfall fermentieren läßt. Dies

31 Giorgio Vasari, Le Vite de’ più eccelenti architetti [...], edizione Firenze 1550, Luciano Bellosi, Aldo Rossi (Hg.), 2 Bde., Torino 1991, hier: Bd. 1, S. 67: Introduzione - Pittura, cap.xx: „E toglievano per quelle tavole i colori ch'erano di miniere, i quali son fatti parte da gli alchimisti e parte trovati nelle cave.“

32 Giulio Campagnola, Padua 1482-ca. 1515; vgl. Eduard A. Safarik: Campagnola, Giulio. In: Dizionario biografico degli italiani, Bd. 17, Roma (Istituto dell'Enciclopedia Italiana) $1974=$ http://www.treccani.it/enciclopedia/giulio-campagnola_(Dizionario-Biografico)/ (eingesehen: 14.06.2019).

33 Hoch wertvoll war und ist der aus Afghanistan stammende Lapislazuli, der noch heute in einem Prozeß von mehr als 40 Arbeitsgängen zur Farbe Ultramarin hergestellt werden muß, davon viele mit denen der Alchemisten korrespondieren; aber auch die preiswertere Alternative, Azurit, war immer noch hinreichend teuer und hieß in der Antike vom Herkunftsort Armenius lapis; zu Augurellis Zeiten wurde er vor allem aus Ungarn importiert. Erst Anfang des achtzehnten Jahrhunderts konnte Azurit zufriedenstellend durch das synthetische Preußischblau ersetzt werden; im fünfzehnten Jahrhundert verwendete man hierzu das sogenannte „künstliche Kupferblau“, auch „blauer Grünspan“ genannt. 
Verfahren wird schon von Plinius überliefert und nach ihm auch mitgeteilt; ${ }^{34}$ allerdings handelt es sich natürlich nicht um Gold, sondern um Kupfer oder Bronze: eben das erwähnte „künstliche Kupferblau“. Dies ist auffällig, wenn es denn richtig ist, daß der Autor der Chrysopoeia zwar wenig von Metallurgie, viel jedoch von der Herstellung der Farbpigmente wisse: warum sollte er da gerade in einer so elementaren Sache irren?

Natürlich kann ein Alchemist, der wie Augurelli der Meinung ist, alle Metalle seien letztlich Abarten von Gold, auch Kupfer Rotgold, flavum aurum, nennen; ${ }^{35}$ es ist aber irreführend, da es in der Aeneis (1,592-593) eindeutig als richtiges Gold verwendet wird, während das erwartete Wort aes alles andere als unpoetisch klänge und hätte gebraucht werden können. Man darf deswegen nicht ausschließen, daß Augurelli hier ein Spiel mit dem Leser treibt, und der Leser zwar vom Grünspan zu hören bekommt, aber an ein ganz anderes Pigment denken soll, ja vielmehr an eine ganze Reihe von Pigmenten in verschiedenen Reinheitsgraden. Als Ersatz für Ultramarin kam beispielsweise Zaffer in Frage, das aus Deutschland importiert in Italien $\mathrm{zu}$ Smalte weiterverarbeitet wurde. ${ }^{36}$ Der Einsatz von Smalte wird schon für die erste Version der Felsgrottenmadonna von Leonardo da Vinci diskutiert, ein Werk, das derzeit in Mailand befindlich sein mußte: da ist es vielleicht nicht zufällig, daß bei allen virgilischen Beitaten die Beschreibung der Grotte der Glaura, in der sich das Urmetall finden solle, von zumindest demselben Bildtypus beeinflußt wird, der höhlenähnlichen Felsgrotte. Wenn Augurelli also vom Grünspan schreibt und seine Herstellung verfälscht, dann soll der Leser allgemein an neue Verfahren zur Herstellung moderner Pigmente denken, deren Technik jedoch nicht verraten werden darf. ${ }^{37}$

34 Viride Hispanicum, aerugo, hier Plin. nat. hist. 34,110-115.

35 Rotes Gold: Aen. 1,592-593. Ähnlich Chrys. 1,98: flavo auro hier Kupfer.

36 Das sogenannte Kobaltblau. Auch dies war ursprünglich ein teurer, aber schon lange bekannter Rohstoff (Cobalt(ii)-oxid), den zu ersetzen es allerdings im späten fünfzehnten Jahrhundert gelang durch die künstlichen Pigmente Zaffer und die daraus hergestellte Smalte. Der Name des ersteren verrät schon den Venezianer Ursprung, denn es handelt sich um eine dialektale Variante des Zaffiro, Saphirs. Seit der Mitte des sechzehnten Jahrhunderts wurden Zaffer wie Smalte vor allem in Venedig hergestellt, aber schon für die erste Version der Felsgrottenmadonna von Leonardo, also um 1485, wird die Verwendung von Smalte diskutiert. Der Felsgrottenmadonna ähnelt Augurellis Beschreibung der Grotte der ,Glaura' sehr: dachte Augurelli konkret an dieses Bild? Allerdings ist die Grottenbeschreibung eine Imitation aus Vergil Georg. 4,333-385 im Aristaeos-Epyllion.

37 Deshalb keine ausdrückliche Erwähnung der Smalte, und deshalb auch ein Fehlen der künstlichen Herstellung des Zinnobers, das eigentlich als Zusammensetzung von Schwefel und Quecksilber hochinteressant für die Arbeit eines Alchemisten hätte sein müssen. Auch 
Hier sind wir an einem Punkt, den Augurelli ausdrücklich thematisiert und auch bejaht: ja, er würde bisweilen mutwillig Falsches schreiben, um die wahre Alchemie zu schützen - hier also Gold statt Bronze wie vorher Gold statt Quecksilber. ${ }^{38}$ Natürlich könnte dies strenggenommen nur für die Goldtransformation im engen Sinne gelten; so dient es der Immunisierung des Alchemisten vor Kritik. Ich nehme aber trotzdem an, dies gilt auch, ja vielmehr vor allem, für Betriebsgeheimnisse der Venezianer Technologie. ${ }^{39}$

Wenn man nämlich überblickt, was Augurelli so en passant an Themen streifte, die sich locker an die Alchemie andocken lassen oder tatsächlich mit ihr in Verbindung stehen, so wird deutlich, daß seine Nachahmung der Georgica Vergils tiefer geht, als zuerst vermutet. ${ }^{40}$ Die vier Bücher der Georgica widmen sich ja nicht einer globalen Landwirtschaftskunde, auch nicht irgendeiner Landwirtschaft. Sie behandeln die ideale Wirtschaft eines idealen Italiens zu einem Zeitpunkt, als Unmengen von Veteranen zu freien Bauern werden sollten. Augurellis Chrysopoeia behandelt nicht irgendwie die Stoffe, welche unter der Erde liegen, noch speziell nur das Opus Magnum. Vielmehr treten, assoziativ, aber auch gedrängt im Exkurs, Spitzentechnologien des Staates Venedig hervor, mit denen er seinen Reichtum und seine Macht begründet. Manche nennt Augurelli offen - so die Glasproduktion von Murano -, manche eher geheimnisbewahrend - so die Farbpigmentindustrie -, und schließlich manche nur am Rande, im Gleichnis etwa wie die Seidenraupe.

Zinnober wurde in Venedig spätestens seit der Mitte des sechzehnten Jahrhunderts industriell im Trockenprozeßverfahren hergestellt; das Verfahren war schon den arabischen Alchemisten bekannt gewesen und kam ursprünglich aus China; vielleicht wurde es zu Augurellis Zeiten in Venedig noch als Betriebsgeheimnis geschützt.

38 Chrys. 3,478-490: „Ac nonnulla etiam (quod parce nos tamen ultro | Fecimus) interdum sparguntur falsa, neque illic | Omnia praecipue credas tibi vera referri, | Ars ubi secretos duci tentatur in usus, | Atque ubi, quo pacto sese experientia promat, | Prodimus addicti perplexae legibus artis. | Quin ubi Naturae vires aperimus, et artem | Illius asseclam decernimus, atque ubi primum | Esse hanc, mox quae ea sit, ostendimus, omnia passim | Vera tibi porro occurrent magis, Et magis inde | Omnia percipies, quae sunt ducenda sub usum, | Quam illinc, artis ubi nos modus ipse doceri | Est visus, nostroque patens fieri documento."

39 Auch bei der Herstellung des Cucurbiten aus spanischer Soda, Ton und Lehm hatte Augurelli an Venezianer Verfahren zur Glasherstellung erinnert. Augurellis offensichtliche Kenntnis von Verfahrenstechniken der Alchemie, die im Unterschied zum Opus Magnum erfolgreich, wiederholbar und gewinnbringend waren, halte ich für den eigentlichen Gegenstand des Werkes, das die Goldtransformation lediglich zum Leitmotiv hat.

40 Anstelle des eigentlich in einer Liste der durch Alchemisten erfundenen Nebenprodukte unverzichtbaren Schwarzpulvers nennt Augurelli in Chrys. 3,329-336 offensichtlich nur eine speziell konstruierte Kanone. 
Aber gerade Letztere zeigt, daß es dem Verfasser um mehr geht als nur ein alchemistisches Lehrgedicht: es ist gleichzeitig, ja vielleicht vornehmlich, Lob seiner Heimatregion in Gestalt ihrer herausragenden Tugend - der Erfindung und Umsetzung von Erfindungen. Diese sieht er nicht in der Instrumentenherstellung - sonst hätte er durchaus die Druckerkunst nennen müssen -, auch nicht im Handel - sonst kämen die Ursprungsorte der Rohstoffe mehr zum Tragen -: es ist also nicht die Stadt Venedig selbst, der sein Interesse vornehmlich gilt, sondern dem unter Venedig stehenden Umfeld, von wo die Produktion erfolgt: die Insel Murano, sicherlich das Gebiet um die Stadt Faenza, nicht zuletzt seine eigene Diözese Treviso. In der Verfahrenstechnik, dem Verwandlungsprozeß von Sand zu Glas, von Bronze zu blauem Pigment - also der Alchemie im weiteren Sinne - liegt Augurellis Interesse. Diesen Prozeß möchte er nicht in den Händen von einfachen Handwerkern sehen, sondern von Spezialisten kontrolliert, die an seiner Optimierung ständig arbeiten sollen.

Der Begriff Alchemist ist ein weitgefaßter; unter ihn fallen alle, die mit für die Alchemie typischen Verfahren - etwa der Destillation - produzieren. Als den reinsten Ausdruck dieser Arbeit hat Augurelli, vielleicht im Rahmen neuplatonischer Spekulationen, die Transformation von unedlem Gold, Quecksilber, zu lauterem Gold gewählt. Das Opus Magnum, Optimum der Optimierung, das möglicherweise nie erreicht werden kann, nicht aber aus den Augen verloren werden darf, ist das Leitmotiv seines Gedichtes, da dies erklärtermaßen der letztliche Zweck aller alchemistischen Forschung ist.

Dieses - im Rahmen der naturwissenschaftlichen Vorstellungen jener Zeit vollkommen realistische - Ziel, seine Arbeitsschritte und seine Arbeitsethik entsprechen dem neuen Typus des Ingenieurs und Verfahrenstechnikers sozusagen emblematisch im Bild des Alchemisten und lassen sich deutlich leichter poetisieren als die jeweils eigenen Schritte zur Herstellung von Kristallglas und Fayence, Schmelzperlen oder Smalte, granuliertem Pulver und dem Scheidewasser. Es steht aber stellvertretend für all die neuen Techniken, gelingt es dem - sozusagen modernen, neuzeitlichen - Alchemisten doch, einen Rohstoff durch eine Reihe von Prozessen, die jeweils nicht durch abergläubische Ingredientien wie faule Eier verunreinigt sind, so $\mathrm{zu}$ veredeln, daß ein gänzlich neuer daraus geworden scheint. Hier sieht Augurelli den Unterschied zu früheren Techniken. Er ist nicht den Unwägbarkeiten eines traditionell überlieferten Prozesses unterworfen, der sich allerdings chemische Prozesse dienstbar machte, die damals noch nicht hätten verstanden werden können. Dagegen ist der neuzeitliche Weg ein innerhalb des physikalischen Modells verständlicher. Zumindest dem Anspruch nach ist der Vorgang überschaubar, wiederholbar und durch ein weiteres Experiment überprüfbar; machbar allerdings wäre er nur, wenn das physikalische Modell stimmte. 
Hier kann auch ein Teil der Erklärung dafür gesucht werden, warum Augurelli sich selbst bewußt der Lüge bezichtigt. $\mathrm{Zu}$ einem gewissen Maß war dies zwar Konventionen der alchemistischen Literatur geschuldet, aber tatsächlich realisierte chemische Prozesse, mit denen die Farbindustrie Venedigs ihre hohen Preise rechtfertigen konnte, dürften einer besonderen Geheimhaltung unterlegen haben. Smalte aus Murano wird, neben der flämischen Smalte, einen besonders hohen Preis erzielt haben; spätestens 1508 ist das Pigment in der Venezianer Malerei nachgewiesen; Venezianer Kaufleute, die Vendecolori, nahmen verschiedene Sorten in ihr Lager und unterschieden zwischen Massenware (peso grosso) und Qualitätsprodukt (peso sottile): ${ }^{41}$ Kein Wunder, wenn ein Dichter wie Augurelli zwar die Produkte kennenlernen konnte, nicht aber Einzelheiten ihrer Herstellung.

Schon daß Augurelli in die Feinheiten eines jeden Prozesses eingeweiht war, dürfte unwahrscheinlich sein; daß er ihn auch noch einem Publikum mitteilen wollte, das hieraus seinen eigenen Gewinn ziehen mochte und den Venezianer Fachleuten ihr Monopol entreißen könnte, wird wohl ausgeschlossen sein. Während das Fachwissen allerdings leicht ersetzt werden konnte durch Mythen, Geheimniskrämerei und antikes, veraltetes Wissen, steigerte der Ersatz auch noch ihren poetischen Wert und schloß das Werk Augurellis noch enger an die Werke Vergils an: wie dieser im sechsten Buch der Aeneis, so kann auch er sich rühmen, aus der Elfenbeinernen Pforte herausgekommen $\mathrm{zu}$ sein.

Aber das sind letztendlich auch nur bekannte, dem antike-affinen Leser liebe Bilder für einen durch sie repräsentierten, zeitgemäßen und ganz neuartigen Zustand: zum ersten den des Stadtstaates Venedig, dessen Schlüsseltechnologien sich herausarbeiten ließen. Zum nächsten einer Technologie der Reinheitsgebote, deren Rezepte auch nachvollziehbar wären, und gerade deshalb geheimnisvoll gehütet werden müssen. Und endlich manifestiert sich hier schon das zaghafte Aufkommen eines Berufstandes, der eigentlich erst im achtzehnten Jahrhundert seinen Siegeszug feiern wird: nicht etwa jener

\footnotetext{
41 Vgl. hierzu Roland Kirschel: Zur Geschichte des Venezianischen Pigmenthandels. Das Sortiment des „Jacobus de Benedictis a Coloribus“. In: Wallraf-Richartz-Jahrbuch, Bd. 63 (2002), 93-158. Louisa C. Matthew: ,Vendecolori a Venezia'. The Reconstruction of a Profession. In: The Burlington Magazine, Bd. 144, No. 1196 (Nov. 2002), 680-686. Julia A. DeLancey: In The Streets Where They Sell Colors: Placing „vendecolori“ in the urban fabric of early modern Venice. In: Wallraf-Richartz-Jahrbuch 72 (2011), S. 193-232.
} 
des theoretischen Chemikers, sondern der des Verfahrenstechnikers und Ingenieurs, der aus organischen Azopigmenten die Industriefarben schaffen wird, aus Erdöl das unzerbrechliche Plasteglas, und dem es schließlich sogar gelingen wird, indem er ganz einfach ein einziges Proton dem Element entnimmt, aus Quecksilber Gold zu machen. ${ }^{42}$

42 Auf Anregung sei noch erwähnt: a) die einzige zweisprachige und kommentierte Ausgabe des Genres zum Thema: Thomas Reiser: Mythologie und Alchemie in der Lehrepik des frühen 17. Jahrhunderts. Die „Chyrseidos Libri IIII“ des Straßburger Dichterarztes Johannes Nicolaus Furichius (1602-1633). Berlin, New York 2011 (Frühe Neuzeit 48); b) Joachim Telle: Alchemie und Poesie. Deutsche Alchemikerdichtungen des 15. bis 17. Jahrhunderts. Untersuchungen und Texte. Mit Beiträgen von Didier Kahn und W. Kühlmann [zur lat. Ld.]. Berlin, Boston 2013. 
